\title{
Research and Development of Digital Educational Products Powered by MARS Simulating Environment
}

\author{
Vyacheslav M. Dmitriev ${ }^{1}$, Taras V. Gandzha ${ }^{1}$, Tatyana N. Zaichenko ${ }^{1 *}$, and Maxim I. \\ Kochergin $^{1}$ \\ ${ }^{1}$ Tomsk State University of Control Systems and Radioelectronics, prosp. Lenina, 40, 634050, \\ Tomsk, Russia
}

\begin{abstract}
Digital learning technologies, virtual laboratories, training models and applications are all essential directions for development of engineering education. This paper discusses the theoretical basis and applications of the Russia-developed MARS automated simulation environment in teaching as a component of digital educational products electronic study guides, virtual and real-virtual laboratories, training illustration modules. Key sections of component model library in MARS are represented. The examples of training illustration modules are discussed. Applying of MARS in learning ensures efficiency of the educational process, as it makes it possible to better organize self-study activities, boosts knowledge acquisition, and makes theoretical study more instructive and accessible.
\end{abstract}

\section{Introduction}

Interactive educational technologies and digital learning technologies, remote and virtual laboratories, training models and applications are all essential directions for development of engineering education [1-4]. Some of the key trends in development of education in global teaching practices include the increased focus on practice and practical content in courses in natural and engineering sciences, as well as the shift of emphasis in teaching towards intellectual development of students and acquisition of competencies associated with practical activities. Modern psychological and pedagogical ideas about the efficient learning process postulate that this process should be organized to primarily rely on independent and active study of the learning content.

Any development efforts in the above directions make it necessary to employ specific methods and tools of simulation. The most effective simulation methodology is the objectoriented methodology. When developing simulation tools, it makes it possible to achieve a higher unification of development and reusability of any modules. In the course of learning, this approach helps develop the skills of object-oriented thinking, which means the ability to identify of elementary objects involved in a process or a task, detail inter-object relations, and describe ways of object manipulations and inter-object interaction.

* Corresponding author: ztntomsk@ rambler.ru 
The most popular simulation software for electrical and electronics engineer are Matlab/Simulink [5-7] and MultiSim [8-11]. Original software is also being developed [12-14]. Among the Russian modeling systems the most famous are MVTU [15], SiminTech [16, 17], PA10 [18]. The software for education applications, based on component circuit method [19] and simulation system MARS [20], is discussed in this article.

\section{Component circuit method and MARS simulation environment}

The component circuit method is designed to support simulation of various objects, including those having a heterogeneous physical nature. The method is based on a formalized representation of the object's structure as a component circuit, which is composed of elementary structural entities - components. The component circuit method is focused on time domain and frequency simulation of devices with lumped parameters.

Component circuit $C$ is a set of objects (see Fig. 1, a):

$$
C=(\mathbf{K}, \mathbf{B}, \mathbf{N}) \text {, }
$$

where $\mathbf{K}$ - a set of components of a circuit; $\mathbf{B}$ - a set of connections (or branches) between all components from $\mathbf{K} ; \mathbf{N}-$ a set of nodes of a circuit, i.e. common points of connections.

Set of components in general includes three components types: sources, converters and measurers for measurements of energy or signals. Component $K_{i}$ may have any quantity $m$ of links $S_{i}, j=l, \ldots m$ (see Fig. 1, b). Each of $S_{j}$ in common case has a pair of topological coordinates: $N_{i}, B_{i}$ - the numbers of a node and a branch; and a pair of variables $\left(V_{n}, V_{b}\right.$ ), where $V_{n}$ - potential, but $V_{b}$ - stream variables. The topological law of equality of potential variables of links, guiding to one and the same node is executed for potential variable. And the low of equality a zero of stream variables of links, guiding to one and the same node is fulfilled for the stream ones. So, for instance, potential variables are: a velocity in the mechanics, voltage in electrotechnics; stream variables are: force, moment of a force and current. In accordance with the relationships variables they named scalar (one variable $\mathrm{Vn}$ is present) and elementary (both $\mathrm{Vn}, \mathrm{Vb}$ are present). A set of any quantity of links of any types is considered as a vector link. Using vector links simplifies a procedure of constructing component circuit. A set of variables corresponds to a vector link. Component circuit may include components with different types of links.

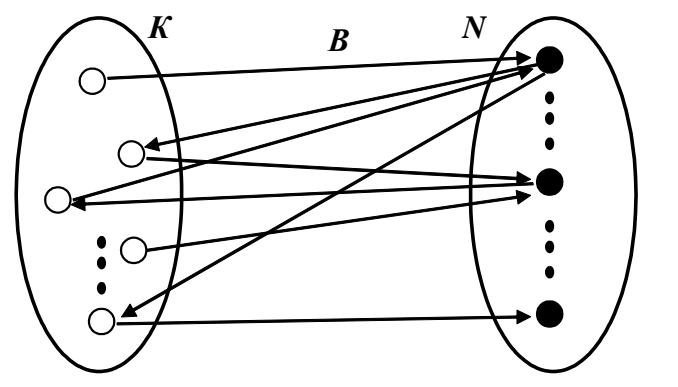

a)

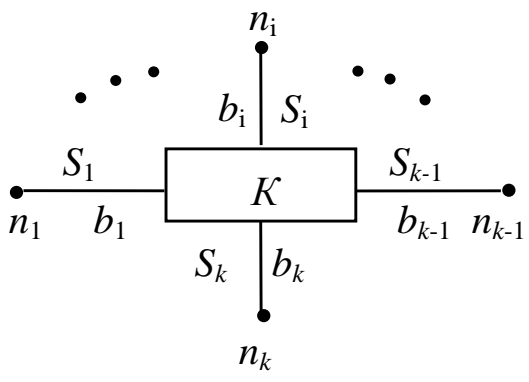

b)

Fig. 1. Component circuit (a) and component (b)

The component circuit model consists of component models and topological laws for potential and stream variables. Mathematical model of the component in common case is a 
set of linear, nonlinear and ordinary differential equations. The solution of a component circuit is a collection all potential and stream variables for all nodes and all branches.

Formalism of the component circuit method allows from the one hand to realize all traditional research methods (structural schemes, electromechanical and etc. analogies), from the other hand - it allows formalized presentation without any analogies.

The component circuit method serves as the theoretical basis for:

- MARS simulation environment (Russian-language abbreviation of "simulation and automated calculation of systems") (MARS SE);

- Macrocalculator system for automation of mathematical calculations;

- experimental tools, both for measurement and control, for real-virtual laboratories.

MARS simulation environment makes it possible to implement a formalized representation and examine the simulated object by means of a computational experiment with a three-level computer model implemented at three levels (layers): object level, logic level, and visual level (see Fig. 2). The object level, which is the layer of the main component circuit in simulation of the object, (C-Layer) is used to formally represent the simulated object; the logical level (L-Layer) is used to define the control algorithms and algorithms for processing of simulated results; the visual level (V-Layer) is used to visualize experimental tools (both for measurement and control).

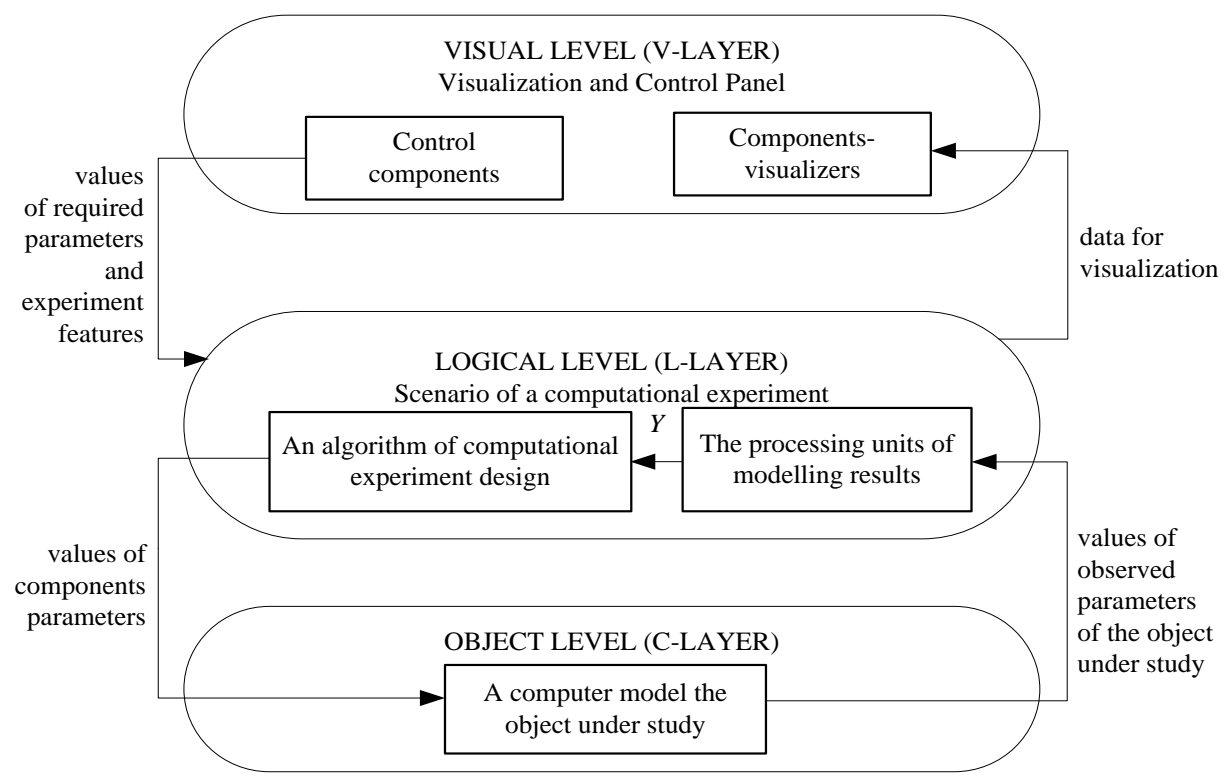

Fig. 2. Structure of a three-level computer model in simulation environment MARS

For the purpose of a computational experiment, MARS SE includes:

- a multi-layer diagram editor allowing the user to graphically design component circuit for the three layers;

- a library of component models, including those of experimental tools (measuring instruments, control and regulation tools) and experimental data processing units;

- computer core that makes it possible to carry out the computational experiment in static and dynamic modes by developing and implementing a mathematical model of the component circuit in the form of a linear algebraic equation system; 
- simulation core designed to simulate algorithms, which are represented as component circuits as well, and based on the message passing mechanism;

- editors of new component models - editors of graphical symbols for all layers, editor of mathematical models.

The process of simulation in MARS SE consists of the following primary steps.

1. Design of the component circuit for the object in the object layer (C-Layer) and its parametrization.

2. Selection of measurable values and setup of measurement tool components in the object layer. Measurement tools set up in the object layer are automatically displayed in the logical layer (L-Layer), which makes it possible to create a component circuit for processing of experimental data.

3. Selection of variable parameters (attributes) of components. This is done using attribute components set up in the logical layer of the model.

4. Setup of the method for variable parameter variation. Variation is achieved either directly using control components in the visual layer (V-Layer), or by means of additional calculations described using algorithms and formulas in the logical layer.

5. Selection of the method for processing of computational experiment results. If necessary, the processing algorithm component circuit is designed in the logical layer (LLayer).

6. Selection of the method for visualization of computational experiment results. Visualization tools are set up in the logical layer and are automatically displayed in the visual layer (V-Layer).

7. Editing of the experimental tool panel (adjustment of component parameters, visualization of results of the computational experiment) in the visual layer ( $\mathrm{V}$-Layer). At this stage, the geometric model of the visual layer is edited - dimensions of measurement tool panels are set and they are placed in the layer window.

8. Selection of the simulation method and parameters.

9. Debugging of the model, including adjustment of the simulation method and parameters.

The main stages of simulation in MARS SE are detailed below.

\section{MARS SE-based virtual laboratories}

Given the universality of the component circuit method, for many years MARS simulation environment has been developing in parallel with research aimed at simulation of objects of various kinds of physical nature. In this regard, a fairly wide range of component models has been implemented and component model libraries have been created for electrical engineering, mechanical engineering, electrical diagram elements, hydraulics, etc. (see Fig. 3).

Interpretation of the statechart diagram into component circuit formalized representation was also realized. Thus simulation of both continuous, discrete and hybrid systems is possible.

That makes it possible to use such models to create virtual training laboratories for a variety of disciplines. In the context of distance learning technologies, such virtual laboratories can become the primary tool in performance of laboratory tasks, and in the context of full-time learning they can be used as a supplementary tool for independent learning that will help students prepare to work with real equipment in specialized university laboratories. Today, virtual training laboratories are used in courses in electrical and electronics engineering, automatic control theory and virtual measurement practice. 


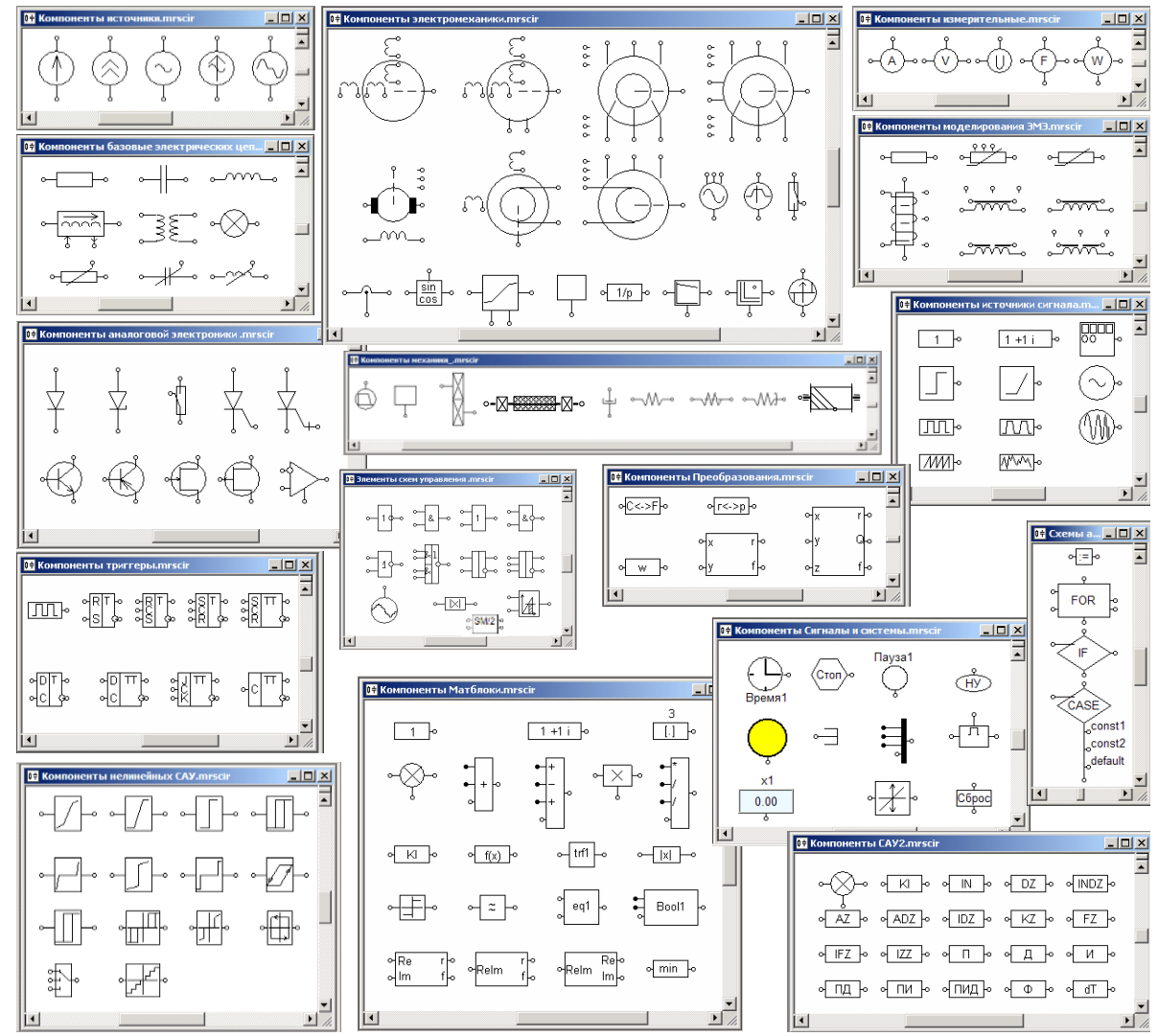

Fig. 3. Key sections of component model library in SE MARS

\section{Training illustration modules}

The purpose of building illustrative modules (TIM) is to illustrate the laws, principles and methods that make up the scope of the discipline. As such, TIM can become an integral part of interactive study guides and manuals.

From the standpoint of simulation and computational experiments, training illustration modules are in fact multi-level models in MARS SE that have been designed and debugged by the instructor or instructional designer. The student's goal primarily is to vary parameters of the model and analyze the results. Design of a TIM follows the same stages as the process of simulation in MARS SE. Once designed and debugged, the TIM is integrated in the interactive study guide (manual) and functions as its element.

Let us consider the stages of building a three-level model in MARS SE using design of a TIM as an example.

\subsection{Example 1. TIM for illustration of Ohm's law and conductor resistance value}

Let it be required to illustrate Ohm's law, the dependence of resistance value in a conductor on its geometric and physical parameters, and calculation of power. Training content will be demonstrated using the example of simulation of an electric circuit that consists of a DC 
voltage source and two resistors (see Fig. 4). The process of TIM design consists of the following steps.

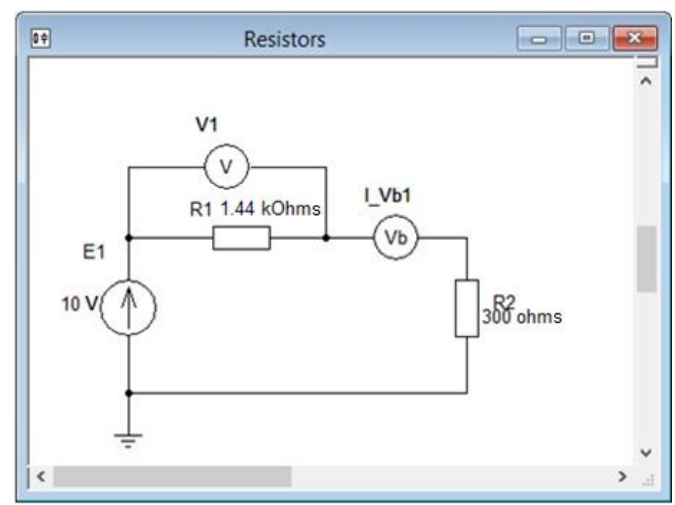

Fig. 4. Object layer of the Ohm's Law TIM

1. A model of the electric circuit is created in the object layer of the circuit editor.

A component circuit is created in the object layer of MARS SE, which includes the DC voltage source E1 and two resistors. Since it is required to demonstrate the dependence of current and voltage on resistance, the voltmeter V1 and the ammeter I_Vb1 are included in the component circuit to perform measurements. They simultaneously transfer the simulation results from the object layer to the logical layer in the editor for further processing (calculation of power) and then to the visual layer for visualization.

2. Parametrization of object-level components and selection of variable parameters.

In this example, R1 resistor resistance is the variable parameter. It will be automatically calculated using the geometric and physical parameters of the conductor specified by the student. The type of the variable parameter is set by the attribute component 1 (see Fig. 5), which is located only in the logical layer in the circuit editor.

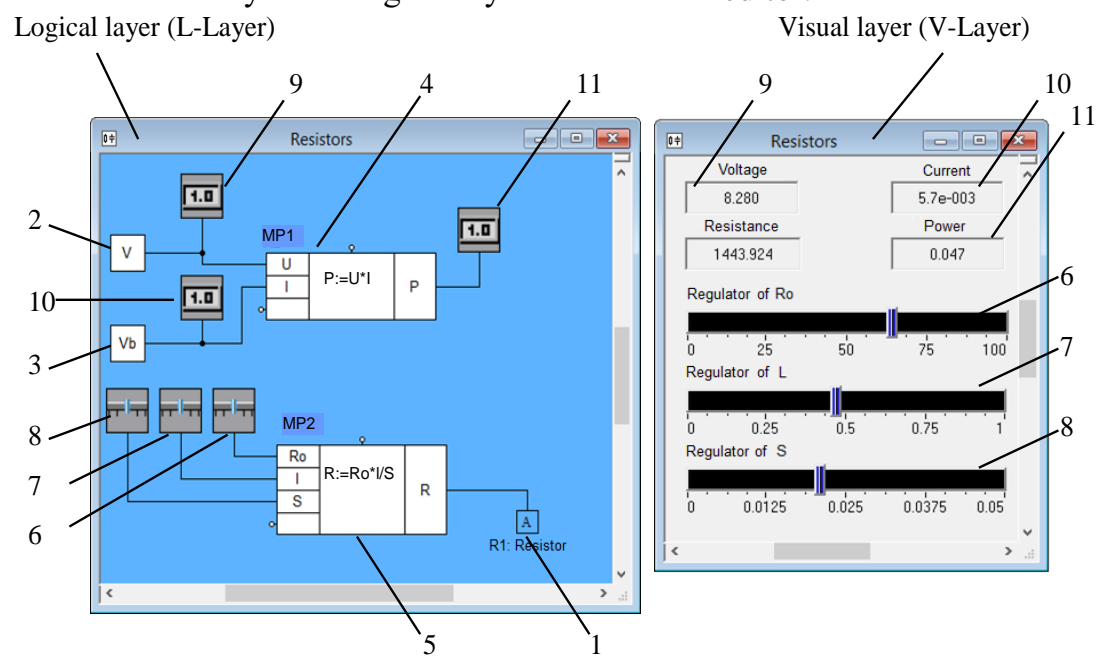

Fig. 5. Logical and visual layers of the Ohm's Law TIM in MARS SE

Resistance variation will be achieved by changing the parameters of the resistor, and resistance value will be calculated automatically. Regulators $6,7,8$ are used to set the specific resistance Ro, length $\mathrm{L}$ and cross-section area $\mathrm{S}$. These regulators are set up in the logical level and automatically displayed in the visual layer. Graphical symbols 
representing the same components in the logical and visual layers in Fig. 4 will be indicated by the same numbers.

3. Creation of the component circuit for calculation of power and resistance.

Mathematical panels 4 и 5 are used to define the formulas for calculation of power and resistance. The voltmeter and ammeter that were earlier set up in the object layer are now automatically displayed in the logical layer as data sources 2 and 3 which are connected to the input of the mathematical panel 4.

4. Multimeters $9-11$ are used to visualize voltage, current and power.

5. Defining the method of setting and adjusting the parameters of the conductor. Parameters will be regulated using regulator components 6-8. They are set up in the logical level and automatically displayed in the visual layer.

Once the resistance is calculated by component 5 using the attribute component 1 , the resistance value is transferred to the object level.

6. Editing the experimental tool panel. Graphical symbols representing experimental tools are placed in the visual layer, their size is adjusted, and as a result the control and measurement tool panel appears as shown in Figure 4.

After the method and parameters of simulation are selected, the model is calculated. Results of calculations are displayed in the visual layer, and parameters are controlled using the regulator components in the visual layer. It should be noted, that the developer of the TIM manages all three layers. The logical layer of a functioning TIM cannot be visualized.

\subsection{Example 2. TIM for study of the laws of mechanics}

One of the simplest dynamic systems, and one of the first ones that students learn, is the physical pendulum. The purpose of the TIM is to demonstrate the effect of the initial displacement, length of the thread, and body mass on the amplitude of oscillations of the pendulum, its velocity, kinetic and potential energy.

A TIM designed for the study of a physical pendulum is shown in Fig. 6 and Fig. 7. The input data (initial displacement angle, length of thread, body mass) is entered and the output data (current linear velocity, kinetic and potential energy) is displayed in the visual layer (see Fig. 6), where the results can also be visualized as graphs. The object layer (C-Layer) is where the mathematical model of the pendulum itself is located (see Fig. 7). In this case, it is represented by a block diagram. The logical layer (L-Layer) is used to calculate the values of the linear velocity and energy of the pendulum. Same as in the previous example, the mathematical model in this TIM is set up using the mathematical panel.

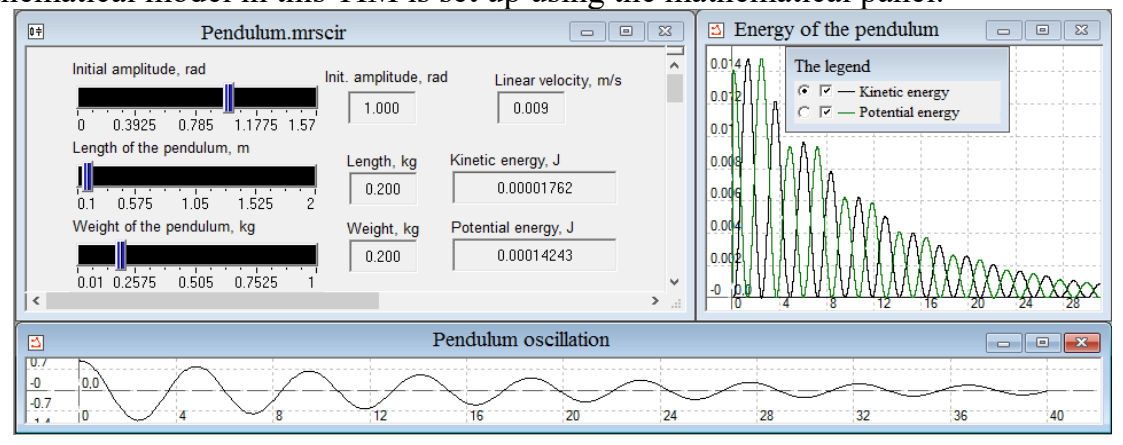

Fig. 6. Visual layer of the Physical Pendulum TIM

Students can vary output parameters of the model and observe changes in the oscillation amplitude of the pendulum, its velocity, kinetic and potential energy. Depending on the competencies desired as the outcome of the course, complexity of the task can be adjusted. 
For example, students can be required to register additional physical effects, which results in modification of the model in the object layer.

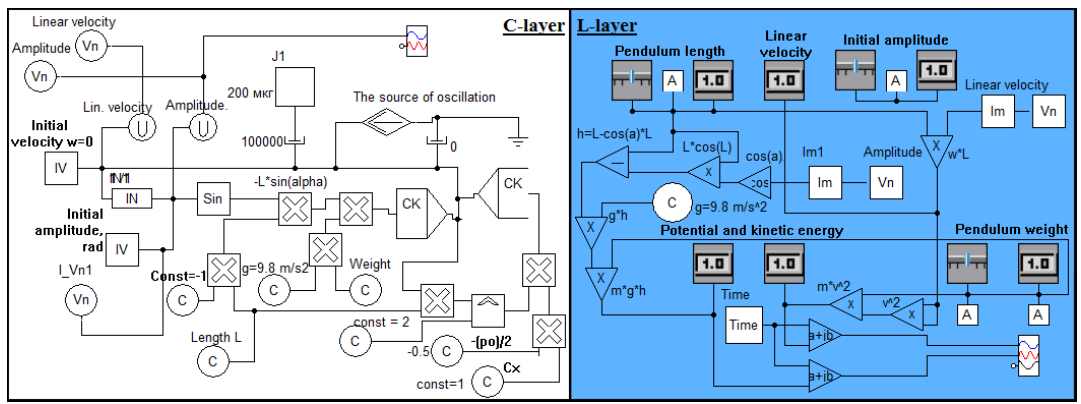

Fig. 7. Object and logical layers of the Physical Pendulum TIM

\section{Conclusion}

MARS simulation environment is used in a variety of academic activities: lectures, practical and laboratory classes, and in independent study. Today, it serves as the basis for virtual laboratories for electrical and electronics engineering, automatic control theory, electrical machinery, system simulation; an interactive tutorial and lecture presentations with training illustration modules on system simulation. Simulation in MARS SE is utilized in project-based group learning, in scientific research and in development of graduate qualification theses. Feedback from students and instructors evidences that the use of MARS SE in learning ensures high efficiency of the process, as it makes it possible to better organize self-study activities, boosts knowledge acquisition in students, and makes theoretical study more instructive and accessible.

\section{References}

1. P. Ptak, Application of software packages in research and didactics, in Proceedings of the International Scientific Conference, Volume II (Vol. 589), p. 597 (2016). doi: 10.17770/sie2016vol2.1418

2. S. Rokooei, J.D. Goedert, L. Najjar, Enhancing Construction Project Management Education by Simulation, in Journal of Professional Issues in Engineering Education and Practice, 143(4), 04017003 (2017). doi: 10.1061/(ASCE)EI.1943-5541.0000329

3. A.J. Magana, Modeling and simulation in engineering education: A learning progression, in Journal of Professional Issues in Engineering Education and Practice, 143(4), 04017008 (2017). doi: 10.1061/(ASCE) EI.1943-5541.0000338

4. P. Litwin, D. Stadnicka, Computer Modeling and Simulation in Engineering Education: Intended Learning Outcomes Development, in International ScientificTechnical Conference MANUFACTURING, pp. 169-184, Springer, Cham (2019). doi: 10.1007/978-3-030-17269-5_12

5. S. Das, Design and Implementation of MATLAB-Simulink Based Solar Cell Modeling and PV System Design Exercises for Advanced Student Learning, in ASEE Annual Conference and Exposition, Conference Proceedings, 2018 June (2018)

6. K. Nehra, V. Nehra, B. Singh, S. Kumar, M. Kumar, Computer simulation using gpsc package matlab, simulink for bioinformatics professional, in ICT Based Innovations, pp. 251-262, Springer, Singapore (2018). doi: 10.1007/978-981-10-6602-3_25 
7. A. Tepljakov, E. Petlenkov, E. A. Gonzalez, I. Petras, Design of a MATLAB-based teaching tool in introductory fractional-order systems and controls, in 2017 IEEE Frontiers in Education Conference (FIE), pp. 1-5, IEEE (2017). doi: 10.1109/FIE.2017.8190681

8. J. Zhang, X.G. Li, Multisim based schematic design and simulation, in Computer Simulation, 5, 032 (2005).

9. K. Dai, S. Zeng, L. Huang, N. Wang, The application of mixed software simulation platform based on Multisim and MATLAB for electric specialty experiment teaching, in 2009 International Conference on Computational Intelligence and Software Engineering, pp. 1-4, IEEE (2009). doi: 10.1109/CISE.2009.5365124

10. S. Mahata, A. Maiti, C.K. Maiti, Cost-effectiveweb-based electronics laboratory using NI MultiSim, LabVIEW and ELVIS II, in 2010 International Conference on Technology for Education, pp. 242-243, IEEE (2010). doi: 10.1109/T4E.2010.5550110

11. B. Heying, D. Kejie, J. Li, Application Multisim to virtual laboratory for experiment teaching, in 2010 International Conference on Computational Intelligence and Software Engineering, pp. 1-4, IEEE (2010). doi: 10.1109/CISE.2010.5676747

12. J. Ospino, M. E. Sánchez, A.R. Secchi, Implementation of a block-oriented model library for undergraduate process control courses in EMSO simulator, in Education for Chemical Engineers, 18, 45-57. (2017). doi: 10.1016/j.ece.2016.08.002

13. N. Ilc, U. Lotrič, FTsim: A 3D Tool for Teaching Automation Concepts, in 2018 13th APCA International Conference on Automatic Control and Soft Computing (CONTROLO), pp. 31-36, IEEE (2018). doi: 10.1109/CONTROLO.2018.8514259

14. D. Draskovic, M. Cvetanovic, B. Nikolic, SAIL-Software system for learning AI algorithms, in Computer Applications in Engineering Education, 26(5), pp. 1195-1216 (2018). doi: 10.1002/cae.21988

15. O.S. Kozlov, L.M. Skvortsov, MVTU software package in scientific research and applied developments, in Mathematical Models and Computer Simulations, 8(4), pp. 358-368. (2016). doi: 10.1134/S2070048216040116

16. F.I. Baum, O.S. Kozlov, I.A. Parshikov, V.N. Petuhov, K.A. Timofeev, A.M. Shchekaturov, SimInTech software for programming control-system devices, in Atomic Energy, 113(6), p. 443 (2013). doi: 10.1007/s10512-013-9660-8

17. About SiminTech. http://help.simintech.ru

18. D.M. Zhuk, V.B. Manichev, M.K. Sakharov, SADEL as a library of "ultra-precise" solvers for the PA10 software package (SADEL-PA10), in MES, pp. 147-153 (2012). doi: 10.1088/1757-899X/630/1/012012

19. E.A. Arais, V.M. Dmitriev, Modelirovaniye neodnorodnykh tsepei i sistem na EVM [Computer modeling of nonhomogeneous circuits and systems], Moscow, Radio i Svyaz (1982)

20. V.M. Dmitriev, A.V. Shutenkov, T.N. Zaichenko, T.V. Gandzha, MARS - sreda kompyuternogo modelirovaniya tekhnicheskikh ustroistv i sistem [MARS - environment for computer modeling of technical devices and systems], Tomsk, V-Spektr (2011) 